\title{
The Influence of Tension on the Success of Aponeurotic Suture of the Anterior Abdominal Wall in Experiment
}

\author{
Yuriy M. Sheptunov, $\mathrm{PhD}, \mathrm{ScD}^{1}$; Pavel V. Vnukov, $\mathrm{PhD}^{2}$; Evgeniy F. Cherednikov, $\mathrm{PhD}, \mathrm{ScD}^{1}$; \\ Andrey A. Filin, $\mathrm{PhD}^{1}$; Evgeniy S. Ovsyannikov, $\mathrm{PhD}^{1}$ \\ ${ }^{1}$ Voronezh State Medical University named after N.N. Burdenko, Voronezh, Russia \\ ${ }^{2}$ Yelets city hospital \#2, Yelets, Russia
}

\begin{abstract}
Background: The problem of predicting the failure of aponeurotic sutures today is of current interest in abdominal surgery, especially in herniology.

Methods and Results: The experimental study was carried out on 20 rabbits of the white giant breed (both sexes). Aponeurotic defects of various sizes were made to the animals' middle zone of the anterior abdominal wall in the area of the anterior rectus sheath. The defects were sutured with a different tension of the aponeurosis depending on the size of the defect. This tension was determined by traction over ligatures conducted through the borders of the reduced aponeurosis by a digital dynamometer where the edges contact. To increase the rigidity of the layers, titanium frames were installed in the retromuscular space. Thus, tension from 0.012 MPa to 1.2 MPa was created. The results were evaluated on the 30th day of the postoperative period Macroscopic assessment under a loupe and histological examination were used. It was found that aponeurotic sutures failed at a higher tension index $(0.66 \pm 0.16 \mathrm{MPa}$ vs. $0.26 \pm 0.16 \mathrm{MPa}, P<0.001)$. At the same time, histological changes were characterized by signs of inflammation with a pronounced alterative component. We did not find that the direction of the incision had any effect on the tension value in cases of suture failure.

Conclusion: No failure of the suture in the early postoperative period was observed in cases of aponeurosis edge tension less than 0.4 MPa. Exceeding this value in $68.7 \%$ of cases led to the failure of aponeurotic sutures. (International Journal of Biomedicine. 2018;8(3):247-249.)
\end{abstract}

Key Words: tension • aponeurosis $\bullet$ abdominal wall $\bullet$ suture failure

\section{Introduction}

Tension of the aponeurosis of the anterior wall is a key concept in abdominal surgery, especially in herniology. Excessive strain on the aponeurosis during suturing is often accompanied by an increase of intra-abdominal pressure; such strain is also the cause of intraoperative and postoperative suture failure, which can lead to eventration and herniation. It is known that about $20 \%$ of laparotomies subsequently lead to the formation of postoperative hernia. ${ }^{(1)}$ There is a large number of methods to reduce the tension on the aponeurosis (separation plastics, introducing botulinum toxin into the

*Corresponding author: Evgeniy S. Ovsyannikov, PhD. Department of faculty therapy, Voronezh State Medical University named after N.N. Burdenko. Voronezh, the Russian Federation. E-mail:ovses@yandex.ru lateral abdominal muscles, applying of different incisions, acute stretching of the transverse abdominal muscle, etc.). ${ }^{(2-4)}$ However, aponeurotic tissue is functionally related to tension; therefore, it is impossible to completely avoid the stretch of aponeurotic edges during an operation or in the postoperative period. To date, it is not known what value of tension is permissible. The only objective criterion, which is used by many authors, is intra-abdominal pressure. ${ }^{(5)}$ At the same time, it often happens that intra-abdominal pressure does not depend on the tension on the aponeurosis.

In surgery, there are different ways to assess the tension in the wound. ${ }^{(6)}$ However, there are no recommendations regarding the thresholds, exceeding which leads to a high degree of probability that aponeurotic suture will fail in the postoperative period. The determination of such thresholds would allow predicting the process of aponeurotic wound healing. 
The purpose of this study was to determine the thresholds for the tension of the edges of aponeurotic wounds, exceeding which leads to the failure of the sutures in the early postoperative period.

\section{Materials and Methods}

The study protocol was reviewed and approved by the Ethics Committee of Voronezh State Medical University named after N.N. Burdenko. All stages of the experiment were carried out in accordance with the requirements of Directive 2010/63/EU of the European Parliament and of the Council of 22 September 2010 on the protection of animals used for scientific purposes.

The study was performed on 20 rabbits of the white giant breed (both sexes). The average weight of the animals was $4.7 \pm 0.41 \mathrm{~kg}(\mathrm{Me}=4.8 \mathrm{~kg})$. Under intravenous anesthesia with thiopental $(25 \mathrm{mg} / \mathrm{kg})$, an incision was made in the skin of the anterior surface of the abdomen, allowing dissection of dissection of the anterior rectus sheaths. Further, a defect of rectangular shape in the aponeurosis was created, symmetrical with respect to the axis of the linea alba of certain sizes (one side of the rectangle was $10 \mathrm{~mm}$, and the second - from $5 \mathrm{~mm}$ to $35 \mathrm{~mm}$, depending on the required tension). Closing the defect (the length of the joined edges of the defect was always equal to $10 \mathrm{~mm}$ ) by bringing the edges of the aponeurosis together created some tension. This tension was determined by traction over ligatures conducted through the borders of the reduced aponeurosis by a digital dynamometer where the edges contact (measurement accuracy of \pm 0.05 Newton $(\mathrm{N})$ ). To calculate the aponeurosis strain in relative value $(\mathrm{MPa})$, the obtained value was divided by the cross-sectional area of the aponeurosis edge. This area is equal to $10 \mathrm{~mm}$ (the size of the reduced edge of the wound) $\times$ the thickness of the aponeurosis. This thickness was determined by a micrometer. In the studied samples, the thickness was $0.2 \pm 0.04 \mathrm{~mm}(\mathrm{Me}=0.2 \mathrm{~mm})$. Taking into account the fact that during the experiment, tension index (TI) was from $0.02 \mathrm{~N}$ to $2.08 \mathrm{~N}(0.8 \pm 0.55 \mathrm{~N})$, the aponeurosis tension in the region of one edge was from $0.012 \mathrm{MPa}$ to 1.2 $\mathrm{MPa}(0.41 \pm 0.29 \mathrm{MPa}, \mathrm{Me}=0.36 \mathrm{MPa})$.

For modeling wound edge tension exceeding $0.72 \mathrm{~N}$, an artificial increase in tissue rigidity was required. It was achieved by installing a rectangular metal frame made of titanium wire in the retro muscular space. This frame, when suturing the aponeurosis in the longitudinal direction with its outer edges resting against the lateral edges of the rectus sheath, created the necessary resistance when the medial edges approached. When suturing the aponeurosis in the transverse direction, the frame created such resistance, by the upper and lower edges resting on the linea alba. The need for such modeling arose because of the pronounced mobility of the layers of the abdominal wall of the rabbit. Since the frame itself was located in the retromuscular space, being almost isolated from the anterior rectus sheath, its influence on the healing of aponeurotic wounds can be ignored.

Thus, 120 aponeurosis defects were formed in laboratory animals, of which 60 were sutured in the longitudinal and 60 in the transverse direction. Closure of the defects was carried out by a continuous polypropylene (thickness 6/0) suture with "bytes" in $2 \mathrm{~mm}$. That is, to suture one defect, it was required to overlap 5-6 stitches of the seam.

The results were evaluated on the 30 th day of the postoperative period because until this time, pronounced infiltrative changes in the wound do not allow assessment of the success of sutures. After euthanasia, macroscopic assessment of the sutures was performed using small magnification $(\times 10)$, as well as microscopy with H\&E staining.

The statistical analysis was performed using the statistical software Microsoft Excel. The mean (M), median (Me) and standard deviation (SD) were calculated. The distribution was estimated by the Kolmogorov-Smirnov criterion with the Lilliefors correction. The Mann-Whitney U Test was used to compare the differences between the two independent groups. The level of statistical significance was 0.01 .

\section{Results}

We did not find a lethal outcome, wound suppuration, or complete eventration with a divergence of cutaneous sutures. In macroscopic assessment under a magnifying glass, it was found that in the area of 87 aponeurotic defects (42 longitudinal and 45 transverse) there was a whitish scar, through which the suture material could be seen. The underlying muscle tissue was not visible. Such wounds are macroscopically evaluated as successful. The aponeurosis TI in such wounds ranged from $0.01 \mathrm{MPa}$ to $0.45 \mathrm{MPa}(0.26 \pm 0.16 \mathrm{MPa})$. Microscopically, we observed in the sections fragments of fibrous adipose tissue with manifestation of minor edema and hyperemia, and a group of transverse-striated muscle fibers of a typical histological structure with mild edema manifestation. Large fragments of fibrous tissue, sometimes with embedded muscle fibers of different thickness with weakly expressed diffusefocal or focal lymph plasma-cell infiltration were observed. There were also fragments of foreign polymer material, surrounded on all sides by fibrous tissue.

During the inspection of 18 wounds, we found a complete divergence of edges of the aponeurosis. The bottom of the wound was either muscle or peritoneum. The aponeurosis $\mathrm{TI}$ in such wounds ranged from $0.49 \mathrm{MPa}$ to $0.98 \mathrm{MPa}(0.72 \pm 0.18 \mathrm{MPa})$. These sutures are determined to be failed. Of these, 9 were in the longitudinal and 9 in the transverse direction. At the same time, fragments of fibrous-fat tissue with signs of edema and vascular hyperemia were found in micro-preparations. Small groups, sometimes bundles of muscle fibers, were observed, in some cases with sites of diffuse, moderately expressed, interstitial inflammation. We also observed fibrous tissue with a few muscle fibers in it, with signs of severe atrophy, and around them pronounced diffuse focal lymph plasma-cell infiltration with moderate, in some cases, neutrophil infiltration.

In the area of 15 wounds, we observed partial divergence of edges of the aponeurosis. In these cases, the underlying muscle tissue was visible through the central part of the scar between the individual stitches. The aponeurosis TI in such wounds ranged from $0.4 \mathrm{M} \mathrm{Pa}$ to $0.68 \mathrm{MPa}(0.57 \pm 0.11 \mathrm{MPa})$. These sutures are determined to be partially failed. Of these, 
9 were in the longitudinal and 6 in the transverse direction. Microscopically, the following changes were observed: fragments of fibro-adipose tissue with symptoms of edema and hyperemia; bundles of muscle fibers, sometimes separated by thin layers of fibrous tissue; large fragments of fibrous tissue with moderate diffuse-focal inflammatory infiltration, sometimes with an admixture of single neutrophils; and a few muscle fibers with signs of atrophy in areas with fibrosis.

In processing the results, it was found that the tension rate in sutures of aponeurosis defects with complete or partial failure have a statistically significant higher value compared to wounds with successful healing $(0.66 \pm 0.16 \mathrm{MPa}$ vs. $0.26 \pm 0.16 \mathrm{MPa}$; $\mathrm{U}=117.0, P<0.001)$.

Partially failed seams also had a statistically significant lower stress value compared to normal sutures $(0.57 \pm 0.11$ MPa vs. $0.72 \pm 0.18 \mathrm{MPa}$; $U=58.5, P<0.01)$. At the same time, we did not observe any influence of the direction of the suture on the tension index that led to a failure of wound healing (U=108, $P=0.338)$.

The obtained data cannot serve as an absolute guide to surgery after recalculation for human aponeurosis. In the postoperative period, due to the verticalization and various reflex and motor acts, tissue tension can vary significantly. ${ }^{(7)}$ However, exceeding the critical values during surgery gives grounds to predict the failure of aponeurotic suture with a high probability.

\section{Conclusion}

During the experimental study, it was found that an increase in the tension of aponeurosis increased the probability of suture failure. No failure of the suture in the early postoperative period was observed in cases of aponeurosis edge tension less than 0.4 MPa. Exceeding this value in $68.7 \%$ of cases led to the failure of aponeurotic suture.

\section{Competing interests} interests.

The authors declare that they have no competing

\section{References}

1. Fink C, Baumann P, Wente MN, Knebel P, Bruckner $\mathrm{T}$, Ulrich $\mathrm{A}$, et al. Incisional hernia rate 3 years after midline laparotomy. Br J Surg. 2014;101(2):51-4. doi:10.1002/ bjs. 9364 .

2. Novitsky YW, Elliott HL, Orenstein SB, Rosen MJ. Transversus abdominis muscle release: a novel approach to posterior component separation during complex abdominal wall reconstruction. Am J Surg. 2012;204(5):709-16. doi: 10.1016/j.amjsurg.2012.02.008.

3. Alam NN, Narang SK, Pathak S, Daniels IR, Smart NJ. Methods of abdominal wall expansion for repair of incisional herniae: a systematic review. Hernia. 2016;20(2):191-9. doi: 10.1007/s10029-016-1463-0.

4. Sheptunov YM, Vnukov PV. Method of prevention of intra-abdominal hypertension in the median ventral hernioplasty. Voronezh State Medical University named after N.N. Burdenko. 2017: 2629803 (25). [In Russian].

5. Muresan M, Muresan S, Bara T, Brinzaniuc K, Sala D, Suciu $\mathrm{B}$, Radu N. The intraabdominal pressure A real indicator of the tension free principle during anterior wall repair procedure after incisional hernias. Ann Ital Chir. 2015;86:421-6.

6. Schachtrupp A, Wetter O, Höer J. An implantable sensor device measuring suture tension dynamics: results of developmental and experimental work. Hernia. 2016;20(4):601-6. doi: 10.1007/s10029-015-1433-y.

7. Vnukov PV, Sheptunov Y.M. Some Strain Characteristics of the White Lne of the Abdomen in the Median Laparotomic Wound (Experimental Study). Vestnik of Experimental and Clinical Surgery. 2016;9(1):76-80. doi: 10.18499/2070-478X2016-9-1-76-80. [Article in Russian]. 